\title{
Discrete analogues of the Dixmier operators
}

\author{
A.E. Mironov
}

\section{Introduction and main results}

In this paper we construct discrete analogues of the Dixmier operators, that is, commuting difference operators corresponding to a spectral curve of genus 1 whose coefficients are polynomials of the discrete variable $n$.

The theories of commuting ordinary differential operators and commuting difference operators have a lot in common, therefore, to begin with, we recall some fundamental results concerning commuting differential operators.

According to the Burchnall-Chaundy Lemma [1], if the two differential operators

$L_{1}=\frac{d^{n}}{d x^{n}}+u_{n-1}(x) \frac{d^{n-1}}{d x^{n-1}}+\ldots+u_{0}(x), L_{2}=\frac{d^{m}}{d x^{m}}+v_{m-1}(x) \frac{d^{m-1}}{d x^{m-1}}+\ldots+v_{0}(x)$

commute, then there exists a nonzero polynomial $Q(\lambda, \mu)$ of two commuting variables $\lambda, \mu$, such that

$$
Q\left(L_{1}, L_{2}\right)=0 \text {. }
$$

On the plane $\mathbb{C}^{2}$ with the coordinates $(\lambda, \mu)$ this polynomial defines the spectral curve

$$
\Gamma=\left\{(\lambda, \mu) \in \mathbb{C}^{2}: Q(\lambda, \mu)=0\right\} .
$$

Suppose that $\Gamma$ be a smooth curve. Considering some common eigen-function $\psi$ of the operators $L_{1}$ and $L_{2}$, one can show that the corresponding eigenvalues $\lambda$ and $\mu$ are connected by a polynomial relation $Q(\lambda, \mu)=0$ (see [2]). Thus, the spectral curve parametrizes the common eigen-functions and eigenvalues of $L_{1}$ and $L_{2}$

$$
L_{1} \psi(x, P)=\lambda \psi(x, P), \quad L_{2} \psi(x, P)=\mu \psi(x, P), \quad P=(\lambda, \mu) \in \Gamma .
$$

The rank of operators $L_{1}, L_{2}$ is called dimension of space of common eigenfunctions with fixed eigenvalues $(\lambda, \mu) \in \Gamma$. 
In the case of operators of rank $l=1$, one can find the function $\psi(x, P)$ (Baker-Akhiezer function) in explicit form by means of the theta-function of the Jacobi variety of the curve $\Gamma$, and in this case the operators' coefficients are easily found. Krichever [2] gives a classification of operators of $\operatorname{rank} l>1$. But the problem of finding the operators' coefficients is not solved in the general case. The following particular results relating to operators of rank more than 1 are known: Dixmier [3] found examples for operators of rank 2 corresponding to an elliptic curve with polynomial coefficients

$$
\begin{gathered}
L_{1}=\left(\frac{d^{2}}{d x^{2}}-x^{3}-\alpha\right)^{2}-2 x \\
L_{2}=\left(\frac{d^{2}}{d x^{2}}-x^{3}-\alpha\right)^{3}-\frac{3}{2}\left(x\left(\frac{d^{2}}{d x^{2}}-x^{3}-\alpha\right)+\left(\frac{d^{2}}{d x^{2}}-x^{3}-\alpha\right) x\right),
\end{gathered}
$$

where $\alpha$ is some constant. Krichever and Novikov [4] found all operators of rank 2 corresponding to the elliptic curve, and Grinevich [5] found spectral data corresponding to operators with rational coefficients. Mokhov [6] found operators for $l=3$, also corresponding to the elliptic curve. In [7] and [8], we found examples for operators with rank 2 corresponding to the curve of genus $g=2$, amongst which there are operators with polynomial coefficients, and also formally self-adjoined operators.

As for smooth operators, for commuting difference operators of the form

$$
L_{1}=\sum_{N_{-}}^{N_{+}} u_{i}(n) T^{i}, L_{2}=\sum_{M_{-}}^{M_{+}} v_{i}(n) T^{i}
$$

where $n \in \mathbb{Z}$ is a discrete variable, and $T$ - the shift operator on the discrete variable

$$
T f(n)=f(n+1),
$$

there exists a spectral curve $\Gamma$ given in $\mathbb{C}^{2}$ by a polynomial $Q(\lambda, \mu)$, parametrizing their common eigen-functions and eigenvalues

$$
L_{1} \psi(n, P)=\lambda \psi(n, P), \quad L_{2} \psi(n, P)=\mu \psi(n, P), \quad P=(\lambda, \mu) \in \Gamma .
$$

Define the rank of operators $L_{1}$ and $L_{2}$ in the same way as for the smooth case as a dimension of space of common eigen-functions in the point in general position $P \in \Gamma$. One of the basic differences between the discrete and the 
smooth case consists of the following. Any commutative ring of ordinary differential operators is isomorphic to a ring of meromorphic functions on the algebraic curve with pole only in the point $Q \in \Gamma$, whereas any commutative ring of difference operators is isomorphic to a ring of meromorphic functions on the algebraic curve with $m$ poles, where $m$ can be any natural number [9]. Such operators are called $m$-point operators.

Mumford [10] and Krichever [11] found spectral data corresponding to two-pointed operators of rank 1.

In this paper we will consider only single-pointed operators. For $l>1$, finding the function $\psi(n, P)$ reduces to solving a Riemann problem, and it can not be found in the explicit form. Krichever and Novikov [9] (see also [4]) suggest a method for finding the operators' coefficients called method of Turin parameters deformation, and this method does not require to find $\psi(n, P)$. They show that the operators' coefficients can be restored from solutions of equations on Turin parameters of holomorphic stable vector bundles that are uniquely given by function $\psi(n, P)$, with the operators' coefficients depending on arbitrary $l$ of functional parameters. Namely, they show that in order to restore the operators' coefficients it suffices to find the matrix function

$$
\chi(n, P)=\Psi(n+1, P) \Psi^{-1}(n, P),
$$

where $\Psi(n, P)$ is a Wronski matrix (see below) built on some basis in the space of common eigen-functions. Applying this method, Krichever and Novikov found operators of rank 2 corresponding to the elliptic curve. Simultaneously, the operators' coefficients are expressed by $\zeta$ and $\wp$-Weierstrass functions of two functional parameters.

In this paper we show spectral data for operators of rank 2 corresponding to the elliptic curve and whose coefficients are expressed by elementary functions of functional parameters. In particular, we find operators that have polynomial coefficients, just like the Dixmier operators.

We can assume the affine part of the spectral curve $\Gamma$ to be given in $\mathbb{C}^{2}$ with the coordinates $(z, w)$ by equation

$$
w^{2}=F(z)=z^{4}+c_{2} z^{2}+c_{1} z+1 .
$$

Take $Q=(0,1) \in \Gamma$ as preferred point. $Q=(0,1) \in \Gamma$ (the ring of commuting difference operators will be isomorphic to a ring of meromorphic functions on $\Gamma$ with pole in $Q$ ). The curve $\Gamma$ allows a holomorphic involution

$$
\sigma: \Gamma \rightarrow \Gamma, \sigma(z, w)=(z,-w)
$$


At $l=2$ the matrice $\chi(n, P), P=(z, w) \in \Gamma$ has the form

$$
\chi(n, P)=\left(\begin{array}{cc}
0 & 1 \\
\chi_{1}(n, P) & \chi_{2}(n, P)
\end{array}\right) .
$$

We consider the case where the involution $\sigma$ does not change $\chi_{1}$, e.g.

$$
\chi_{1}(n, P)=\chi_{1}(n, \sigma(P)), P \in \Gamma .
$$

Then, the following holds:

Theorem 1 The functions $\chi_{1}(x, P) u \chi_{2}(x, P)$ have the form

$$
\begin{gathered}
\chi_{1}(n, P)=\frac{c(n)}{z-\gamma(n)}+\frac{c(n)}{\gamma(n)-\gamma(n+1)}, \\
\chi_{2}(n, P)=\frac{1}{2 z}+\frac{a(n)}{2(z-\gamma(n))}+\frac{w \gamma(n)}{2 z(\gamma(n)-z)}+d(n),
\end{gathered}
$$

where

$$
\begin{gathered}
c(n)=\frac{\gamma(n-1)\left(a^{2}(n)-F(\gamma(n))\right.}{4 \gamma(n)(\gamma(n)-\gamma(n-1))} \\
d(n)=\frac{(a(n+1)-1) \gamma(n)+(a(n)+1) \gamma(n+1)}{2(\gamma(n)-\gamma(n+1)) \gamma(n+1)},
\end{gathered}
$$

$\gamma(n), a(n)$ are arbitrary functions of the discrete variable $n \in \mathbb{Z}$.

The function $\lambda_{1}(z)$ on the curve $\Gamma$, with the only second-order pole in $Q$, looks as follows:

$$
\lambda_{1}=\frac{1}{2 z^{2}}+\frac{c_{1}}{4 z}+\frac{w}{2 z^{2}} .
$$

Let $b_{i}(n), e_{i}(n), p_{i}$ denote the series decomposition coefficients of functions $\chi_{1}, \chi_{2}$, and $\lambda_{1}$ in the neighbourhood of $Q$ :

$$
\begin{gathered}
\chi_{1}(n, z)=b_{0}(n)+b_{1}(n) z+\ldots, \quad \chi_{2}(n, z)=\frac{1}{z}+e_{0}(n)+e_{1}(n) z+\ldots, \\
\lambda_{1}=\frac{1}{z^{2}}+\frac{p_{1}}{z}+p_{0}+\ldots, \quad p_{0}=-\frac{c_{1}^{2}}{16}+\frac{c_{2}}{4}, \quad p_{1}=\frac{c_{1}}{2} .
\end{gathered}
$$

The coefficients $b_{i}, e_{i}, i=0,1$ are expressed by $\gamma(n)$, and $a(n)$ by formulae $(6)-(9)$. 
Corollary 1 The operator $L\left(\lambda_{1}\right)$ has the following form

$$
L\left(\lambda_{1}\right)=T^{2}+u_{1}(n) T+u_{0}(n)+u_{-1}(n) T^{-1}+u_{-2}(n) T^{-2},
$$

where

$$
\begin{gathered}
u_{1}(n)=p_{1}-e_{0}(n)-e_{0}(n+1), \\
u_{0}(n)=p_{0}-b_{0}(n)-b_{0}(n+1)-p_{1} e_{0}(n)+e_{0}^{2}(n)-e_{1}(n)-e_{1}(n+1), \\
u_{-1}(n)=-b_{1}(n)+b_{0}(n)\left(-p_{1}-\frac{b_{1}(n-1)}{b_{0}(n-1)}+e_{0}(n-1)+e_{0}(n)\right) \\
u_{-2}(n)=b_{0}(n) b_{0}(n-1) .
\end{gathered}
$$

The basic result of this work consists of the following:

Theorem 2 If we put the function parameters $a(n), \gamma(n)$ equal to

$$
a(n)=n+1, \gamma(n)=n,
$$

then the operators will have coefficients polynomial on $n$. At the same time,

$$
\begin{gathered}
L_{2}=T^{2}+2(n+2) T-\left(\frac{n^{4}}{2}+n^{3}-\frac{1}{2}\left(1-c_{2}\right) n^{2}-\frac{1}{2}\left(8-c_{1}-c_{2}\right) n\right)- \\
-\frac{1}{2}\left(n^{3}+\left(c_{2}-1\right) n+c_{1}-2\right)\left(n^{2}+n-1\right) T^{-1}+ \\
\frac{1}{16}\left(n^{3}+\left(c_{2}-1\right) n+c_{1}-2\right)\left(n^{3}-3 n^{2}+\left(2+c_{2}\right) n+c_{1}-c_{2}-2\right)(n+1)(n-2) T^{-2} .
\end{gathered}
$$

In Theorem 2 we do not list the third order operator due to its bulkiness. Let's look at an example. Let a spectral curve be given by the equation

$$
w^{2}=z^{4}+z^{2}+1
$$

then

$$
\begin{aligned}
L_{2}=T^{2}+2(n+ & 2) T-\frac{1}{2}\left(n^{4}+2 n^{3}-7 n-5\right)-\frac{1}{2}\left(n^{3}-2\right)\left(n^{2}+n-1\right) T^{-1} \\
& +\frac{1}{16}\left(n^{3}-3 n^{2}+3 n-3\right)(n+1)(n-2) T^{-2}, \\
L_{3}= & T^{3}+\left(3 n+\frac{15}{2}\right) T^{2}-\frac{3}{4}\left(n^{4}+4 n^{3}+5 n^{2}-8 n-14\right) T
\end{aligned}
$$




$$
\begin{gathered}
-\frac{3}{4}\left(2 n^{5}+7 n^{4}+10 n^{3}+n^{2}-12 n-5\right) \\
+\frac{3}{16}\left(n^{8}-2 n^{6}-12 n^{5}-3 n^{4}+10 n^{3}+20 n^{2}+6 n-12\right) T^{-1} \\
+\frac{3}{32} n\left(2 n^{2}-n-5\right)\left(n^{6}-3 n^{5}+3 n^{4}-5 n^{3}+6 n^{2}-6 n+6\right) T^{-2}- \\
\frac{1}{64}(n-3)\left(n^{2}-1\right)\left(n^{3}-2\right)\left(n^{3}-6 n^{2}+12 n-10\right)\left(n^{3}-3 n^{2}+3 n-3\right) T^{-3} .
\end{gathered}
$$

In [7] and [8], we use an analogue of reduction (1) in the smooth case in order to find commuting differential operators of rank 2, genus 2 .

In section 2 we recall the Krichever-Novikov equation [9] on the discrete Turin parameters dynamics and give formulae for coefficients of operators of

rank 2 , genus 1 that are expressed by $\zeta$ and $\wp$-Weierstrass functions.

In section 3 , theorems 1 and 2 are proven.

\section{Krichever-Novikov equations on the discrete dynamic of the Turin parameters}

As noted above, for $l>1$ function $\psi(n, P)$ cannot be found in its explicit form. Let $\Psi(n, P)$ denote a Wronski matrice $\Psi^{i j}(n, P)=\psi^{j}(n+i)$, where $\psi^{j}(n, P), 1 \leq j \leq l$ is some basis in the space of common eigen-functions. As shown in [9], the number of zeros of functions $\operatorname{det} \Psi(n, P)$ equals $l g$, where $g$ is the genus of $\Gamma$. Denote them by $\gamma_{1}(n), \ldots, \gamma_{l g}(n)$. By $\alpha_{j}(n)$ we denote vectors where

$$
\alpha_{j}(n) \Psi\left(n, \gamma_{j}(n)\right)=0 .
$$

These vectors are defined up to proportionality. The set $\left(\gamma_{j}(n), \alpha_{j}(n)\right)$ is called Turin parameters. The Turin parameters uniquely define a stable holomorphic bundle of rank $l$ on the curve $\Gamma$. The following theorem, proven in [9], defines the discrete dynamics of Turin parameters.

- The matrix function $\chi(n, P)$ has simple poles in points $\gamma_{j}(n)$. The relations on the residues of the matrix elements

$$
\alpha_{s}^{j} \operatorname{Res}_{\gamma_{s}(n)} \chi^{m i}(n, P)=\alpha_{s}^{i}(n) \operatorname{Res}_{\gamma_{s}(n)} \chi^{m j}(n, P)
$$

do hold. 
The points $\gamma_{s}(n+1)$ are zeros of the matrix determinant $\chi(n, P)$, e.g.

$$
\operatorname{det} \chi\left(n, \gamma_{s}(n+1)\right)=0 \text {. }
$$

The vector $\alpha_{j}(n+1)$ satisfies the equation

$$
\alpha_{j}(n+1) \chi\left(n, \gamma_{j}(n+1)\right)=0 .
$$

Except for the poles $\gamma_{j}(n)$ one of the components $\chi$ has a simple pole in the preferred point $Q$.

As follows from the Riemann-Roch theorem, $\chi(n, P)$ is uniquely restored by the Turin parameters $\left(\gamma_{j}(n), \alpha_{j}(n)\right)$ and by $l$ arbitrary functional parameters.

This theorem enables us to find operators of rank 2 corresponding to an elliptic curve. Below, we give the according theorem, for simplicity only for the case where a holomorphic involution on an elliptic curve switches the location of the poles of function $\chi_{1}$. We give the elliptic curve $\Gamma$ as a factor $\mathbb{C} / \Lambda$, where $\Lambda$ is some lattice in $\mathbb{C}$ and let $z$ be a coordinate in $\mathbb{C}$. Assume that points $\gamma_{1}(n)$ and $\gamma_{2}(n)$ are interchanged by a holomorphic involution on $\Gamma$

$$
\sigma\left(\gamma_{1}(n)\right)=\gamma_{2}(n)
$$

where $\sigma(z)=-z$. The following theorem was proven in [9].

- The operator corresponding to function $\wp(z)$ has the form

$$
L=L_{2}^{2}-\wp(\gamma(n))-\wp(\gamma(n-1)),
$$

where

$$
\begin{gathered}
L_{2}=T+v(n)+c(n) T^{-1}, \\
c(n+1)=\frac{1}{4}\left(s^{2}(n)-1\right) F(\gamma(n+1), \gamma(n)) F(\gamma(n-1), \gamma(n)), \\
v(n+1)=\frac{1}{2}(s(n) F(\gamma(n+1), \gamma(n))-s(n+1) F(\gamma(n), \gamma(n+1)), \\
F(u, v)=\zeta(u+v)-\zeta(u-v)-2 \zeta(v),
\end{gathered}
$$

$s(n), \gamma(n)$ are arbitrary functional parameters.

As can be seen from the formulae for the coefficients of operator $L$, the coefficients are expressed by function parameters $s(n)$ and $\gamma(n)$ applying the $\wp$ and $\zeta$-Weierstrass functions. In corollary 1 and theorem 2 we found conditions for these coefficients being elementary functions and, in particular, polynomials. 


\section{Proof of theorems 1 and 2}

As follows from our assumption on the invariance $\chi_{1}(n, P), P=(z, w) \in \Gamma$ under the action of involution $\sigma, \chi_{1}(n, P)$ has the form

$$
\chi_{1}(n, P)=\frac{c(n)}{z-\gamma(n)}+d_{0}(n)
$$

where $c(n), \gamma(n)$ and $d_{0}(n)$ are some functions of a discrete variable. Note that function $\chi_{1}(n, P)$ has poles in

$$
P(n)=(\gamma(n), \sqrt{F(\gamma(n)}), \quad \sigma P(n)=(\gamma(n),-\sqrt{F(\gamma(n)}) .
$$

From equalities (4), being equivalent in our case to

$$
\chi_{1}(n, P(n+1))=\chi_{1}(n, \sigma P(n+1))=0
$$

get

$$
\chi_{1}(n, P)=\frac{c(n)}{z-\gamma(n)}+\frac{c(n)}{\gamma(n)-\gamma(n+1)} .
$$

Look for function $\chi_{2}$ in the form

$$
\chi_{2}(n, P)=\frac{1}{2 z}+\frac{a(n)}{2(z-\gamma(n))}+\frac{w \gamma(n)}{2 z(\gamma(n)-z)}+d(n),
$$

where $a(n)$ and $d(n)$ are some functions. Note that $\chi_{2}(n, P)$ has poles in points $P(n), \sigma P(n)$ and $Q$, moreover $\operatorname{Res}_{Q} \chi_{2}=1$.

Vectors $\alpha_{1}$ and $\alpha_{2}$ are defined up to proportionality, therefore we can assume them to have the form

$$
\alpha_{1}(n)=\left(a_{1}(n), 1\right), \alpha_{2}(n)=\left(a_{2}(n), 1\right)
$$

Then from (5) we get

$$
\begin{gathered}
a_{1}(n)=-\chi_{2}(n-1, P(n))= \\
\frac{1}{2 \gamma(n)}+\frac{a(n-1)}{2(\gamma(n)-\gamma(n-1))}+\frac{\sqrt{F(\gamma(n))} \gamma(n-1)}{2 \gamma(n)(\gamma(n-1)-\gamma(n))}+d(n-1), \\
a_{2}(n)=-\chi_{2}(n-1, \sigma P(n))=
\end{gathered}
$$




$$
\frac{1}{2 \gamma(n)}+\frac{a(n-1)}{2(\gamma(n)-\gamma(n-1))}-\frac{\sqrt{F(\gamma(n))} \gamma(n-1)}{2 \gamma(n)(\gamma(n-1)-\gamma(n))}+d(n-1) .
$$

Further, the residues of functions $\chi_{1}$ and $\chi_{2}$ in poles $P(n)$ and $\sigma P(n)$ are equal to

$$
\begin{gathered}
\operatorname{Res}_{P(n)} \chi_{1}=\operatorname{Res}_{\sigma P(n)} \chi_{1}=c(n), \\
\operatorname{Res}_{P(n)} \chi_{2}=\frac{1}{2}(a(n)-\sqrt{F(\gamma(n)}), \\
\operatorname{Res}_{\sigma P(n)} \chi_{2}=\frac{1}{2}(a(n)+\sqrt{F(\gamma(n)}) .
\end{gathered}
$$

From equalities (3), in our case taking the form

$$
\begin{gathered}
\operatorname{Res}_{P(n)} \chi_{1}=a(n) \operatorname{Res}_{P(n)} \chi_{2}, \\
\operatorname{Res}_{\sigma P(n)} \chi_{1}=a(n) \operatorname{Res}_{\sigma P(n)} \chi_{2},
\end{gathered}
$$

get

$$
\begin{gathered}
c(n)=\frac{\gamma(n-1)\left(a^{2}(n)-F(\gamma(n))\right.}{4 \gamma(n)(\gamma(n)-\gamma(n-1)}, \\
d(n)=\frac{(a(n+1)-1) \gamma(n)+(a(n)+1) \gamma(n+1)}{2(\gamma(n)-\gamma(n+1)) \gamma(n+1)} .
\end{gathered}
$$

Thus, theorem 1 is proven.

Now find the coefficients of operator $L_{2}$ as follows. Express $\psi(n+2, P)$ and $\psi(n-2, P)$ by $\psi(n-1, P), \psi(n, P), \chi_{1}$ and $\chi_{2}$. For this use the identity

$$
\psi(n+1)=\psi(n-1) \chi_{1}(n)+\psi(n) \chi_{2}(n)
$$

from which

$$
\begin{gathered}
\psi(n+2)=\psi(n-1) \chi_{1}(n) \chi_{2}(n+1)+\psi(n)\left(\chi_{1}(n+1)+\chi_{2}(n) \chi_{2}(n+1)\right), \\
\psi(n-2)=-\psi(n-1) \frac{\chi_{2}(n-1)}{\chi_{1}(n-1)}+\frac{\psi(n)}{\chi_{1}(n-1)} .
\end{gathered}
$$

Now replace $T^{2} \psi(n)$ and $T^{-2} \psi(n)$ in equation

$$
L_{2} \psi=\left(T^{2}+u_{1}(n) T+u_{0}(n)+u_{-1} T^{-1}+u_{-2} T^{-2}\right) \psi(n)=\lambda_{1} \psi(n)
$$

by corresponding expressions. Get

$$
P_{1}(n, P) \psi(n, P)+P_{2}(n, P) \psi(n-1, P)=\lambda_{1} \psi(n, P),
$$


where

$$
\begin{gathered}
P_{1}(n)=\chi_{1}(n+1)+\chi_{2}(n+1) \chi_{2}(n)+u_{1}(n) \chi_{2}(n)+u_{0}(n)+\frac{u_{-2}(n)}{\chi_{1}(n-1)}, \\
P_{2}(n)=\chi_{2}(n+1) \chi_{1}(n)+u_{1}(n) \chi_{1}(n)+u_{-1}(n)-u_{-2}(n) \frac{\chi_{2}(n-1)}{\chi_{1}(n-1)} .
\end{gathered}
$$

In the space of common eigen-functions of operators $L_{2}$ and $L_{3}$ we can chose a basis $\psi_{1}$ and $\psi_{2}$ normalized by conditions

$$
\psi_{1}\left(n_{0}, P\right)=1, \psi_{2}\left(n_{0}, P\right)=0,
$$

with functions $\chi_{1}, \chi_{2}$ not depending on normalization points $n_{0}$ (see [9]), consequently, we have the identities

$$
P_{1}(n, P)-\lambda_{1}=0, \quad P_{2}(n, P)=0 .
$$

Now, having found in theorem 1 the functions $\chi_{1}$ and $\chi_{2}$, find the coefficients $u_{i}(n)$ of operator $L_{2}$ from the series decomposition $P_{1}-\lambda_{1}$ and $P_{2}$ in the neighbourhood of point $Q$.

The series decomposition coefficients $b_{j}, e_{j}$ of functions $\chi_{1}$ and $\chi_{2}$ in the neighbourhood of $Q$ are expressed by the functional parameters $a(n)$ and $\gamma(n)$ according to formulae

$$
\begin{gathered}
b_{0}(n)=\frac{\gamma(n-1) \gamma(n+1)\left(F(\gamma(n))-a^{2}(n)\right)}{4(\gamma(n-1)-\gamma(n))(\gamma(n)-\gamma(n+1)) \gamma(n)^{2}}, \\
b_{1}(n)=\frac{\gamma(n-1)\left(a^{2}(n)-F(\gamma(n))\right.}{4(\gamma(n-1)-\gamma(n)) \gamma^{3}(n)} \\
e_{0}(n)=\frac{1}{2}\left(\frac{c_{1}}{2}+\frac{1}{\gamma(n)}-\frac{a(n)}{\gamma(n)}+\right. \\
\left.\frac{(a(n+1)-1) \gamma(n)+(a(n)+1) \gamma(n+1)}{(\gamma(n)-\gamma(n+1)) \gamma(n+1)}\right) \\
e_{1}(n)=\frac{8-8 a(n)+4 c_{1} \gamma(n)-\left(c_{1}^{2}-4 c_{2}\right) \gamma^{2}(n)}{16 \gamma^{2}(n)} .
\end{gathered}
$$

Analogically, find the operator corresponding to a meromorphic function with one third order pole in $Q$. Direct verification yields that for

$$
a(n)=n+1, \gamma(n)=n
$$


the operators' coefficients are polynomials on $n$. Thus, theorem 2 is proven.

\section{References}

[1] Burchnall, J.L.; Chaundy, I.W. Commutative ordinary differential operators // Proc. London Math. Society. 1923. Ser. 2. V. 21. P. 420-440.

[2] Krichever, I.M. Commutative rings of ordinary differential operators // Funk. Anal. Pril. 1978. V. 12. N. 3. P. 20-31.

[3] Dixmier, J. On Wiyl algebras // Matematika. 1969. V.13, N. 4. P. $16-44$.

[4] Krichever, I.M.; Novikov S.P. Holomorphic vector bundles on Riemann surfaces and the Kadomtcev-Petviashvili equation // Funk. Anal. Pril. 1978. V. 12. N. 4. P. 41-52.

[5] Grinevich, P.G. Rational solutions of the commutativity equation of differential operators. // Funk. Anal. Pril. 1982. V. 16. N. 1. P. 19-24.

[6] Mokhov, O.I. Commuting differential operators of rank 3, and nonlinear equations// Izv. Akad. Nauk SSSR Ser. Matem. 1989. V. 53. N. 6, P. 1291-1314.

[7] Mironov, A.E. A ring of commuting differential operators of rank 2 corresponding to a curve of genus 2// Sbornik Mathematics. 2004. V. 195. N. 5. P. 711-722.

[8] Mironov, A.E. Commuting Rank 2 differential operators corresponding to a curve of genus $2 / /$ Funct. Anal. and its Appl. 2005. V. 39. N. 3. P. 240-243.

[9] Krichever, I. M., Novikov, S. P. A two-dimensionalized Toda chain, commuting difference operators, and holomorphic vector bundles // Russ. Math. Surveys. 2003. V. 58, N. 3, P. 473-510

[10] Mumford, D. An algebro-geometric construction of commuting operators and of solution to the Toda lattice equation, Kortweg-de Vries equation and related nonlinear equations // Proceedings of the International Symposium on Algebraic Geometry (Kyoto Univ., Kyoto, 1977). Tokyo: Kinokuniya Book Store, 1978. P. 115-153.

[11] Krichever, I. M. Algebraic curves and nonlinear difference equations // Uspekhi Matem. Nauk. 1978. V. 33, N. 4, P. 215-216.

Sobelev Institute of Mathematics, 630090 Novosibirsk, Russia; E-mail: mironov@math.nsc.ru 